\title{
Treino Funcional de Marcha no Ambiente Aquático em Pacientes Pós Acidente Vascular Cerebral
}

\author{
Functional gait training in the aquatic environment in post- \\ stroke patients
}

\section{Entrenamiento funcional de la marcha en el medio acuático en pacientes después de un accidente cerebrovascular}

\author{
José Luis Rodrigues Barbosa ${ }^{1}$, Daniela Ramos de Campos Magalhães², \\ Flavia Aparecida de Lima33, Douglas Martins Braga ${ }^{4}$
}

1.Fisioterapeuta, especialista em fisioterapia neurológica - UMESP, fisioterapeuta do setor da fisioterapia aquática da AACD - Ibirapuera, São Paulo-SP, Brasil. https://orcid.org/0000-0002-3321-6241

2.Fisioterapeuta, aprimoramento em reabilitação física com enfoque multidisciplinar - $A A C D$, fisioterapeuta do setor da fisioterapia aquática da AACD - Ibirapuera, São Paulo-SP, Brasil. https://orcid.org/0000-0001-6214-7719

3. Fisioterapeuta, aperfeiçoando em Fisioterapia nas disfunções neurológicas da criança e do adulto - AACD - Ibirapuera, São Paulo-SP, Brasil. https://orcid.org/0000-0001-9580-4471

4.Fisioterapeuta, Mestre em ciências da saúde - UNIFESP, coordenador de fisioterapia do serviço de fisioterapia aquática da AACD, São Paulo-SP, Brasil. https://orcid.org/0000-0001-7293-6754

\begin{abstract}
Resumo
Introdução. Indivíduos após Acidente Vascular Cerebral (AVC) participam menos de atividade física, gerando déficit funcional em habilidades como sentar, levantar e qualidade da marcha, promovendo impactos na qualidade de vida desses indivíduos. Objetivo. Avaliar aspectos relacionados à marcha, equilíbrio e velocidade em pacientes pós AVC após treino funcional de marcha em ambiente aquático. Método. Foram selecionados 4 pacientes com diagnóstico de AVC, randomizados em dois grupos: grupo experimental (GE) e grupo controle (GC), o GE realizou treino funcional de marcha em ambiente aquático e o GC realizou Fisioterapia aquática convencional. Resultados. Houve melhora $(p<0,05)$ nos resultados do Teste Caminhada de 10 metros, Mini Bestest e Time Up and Go. Conclusão. O protocolo de treino funcional de marcha em ambiente aquático teve influência positiva sobre os aspectos relacionados à marcha, em especial o equilíbrio e velocidade da marcha em paciente pós AVC.
\end{abstract}

Unitermos. Hidroterapia; Acidente Vascular Encefálico; Marcha; Fisioterapia

\begin{abstract}
Introduction. Individuals after stroke participate less in physical activity, generating functional deficit in skills such as sitting, standing and gait quality, promoting impacts on the quality of life of these individuals. Objective. To evaluate aspects related to gait, balance, and speed in post stroke patients after functional gait training in an aquatic environment. Method. 4 patients diagnosed with strokes were selected, randomized into two groups: experimental group (EG) and control group (CG), the EG performed functional gait training in an aquatic environment, and the CG performed conventional aquatic physical therapy. Results. There was an improvement $(p<0.05)$ in the results of the 10 -meter walk test, Mini Bestest and Time Up and Go. Conclusion. The functional gait training protocol in an aquatic environment had a positive influence on gait-related aspects, especially the balance and speed of gait in post stroke patients.
\end{abstract}

Keywords. Hydrotherapy; Stroke; Gait; Physical Therapy 


\section{Resumen}

Introducción. Los individuos después de un accidente cerebrovascular participan menos en la actividad física, lo que genera un déficit funcional en habilidades tales como la calidad de sentarse, pararse y andar, lo que promueve impactos en la calidad de vida de estos individuos. Objetivo: Evaluar los aspectos relacionados con la marcha, el equilibrio y la velocidad en los pacientes después de un entrenamiento funcional de la marcha en un entorno acuático. Método. Se seleccionaron 4 pacientes diagnosticados con accidentes cerebrovasculares, asignados al azar a dos grupos: grupo experimental (GE) y grupo de control (GC), el GE realizó un entrenamiento funcional de la marcha en un entorno acuático, y el GC realizó una fisioterapia acuática convencional. Resultados. Hubo una mejora $(p<0,05)$ en los resultados de la prueba de caminata de 10 metros, el Mini Bestest y Time Up and Go. Conclusión. El protocolo de entrenamiento funcional de la marcha en un entorno acuático influyó positivamente en los aspectos relacionados con la marcha, especialmente el equilibrio y la velocidad de la marcha en los pacientes después de un accidente cerebrovascular.

Palabras clave. Hidroterapia; Accidente Cerebrovascular; Marcha; fisioterapia

Trabalho realizado no setor de fisioterapia aquática da Associação de Assistência à Criança Deficiente AACD Ibirapuera, São Paulo-SP, Brasil.

\section{INTRODUÇÃO}

O acidente vascular cerebral (AVC) lidera as causas de incapacidade mundialmente, constituindo um grupo de distúrbios neurológicos com etiologias e quadros clínicos diversificados, resultando na maioria das vezes em alterações motoras, sensoriais, perceptuais, cognitivas e comportamentais ${ }^{1-3}$. Estima-se que, após 6 meses do evento, $26 \%$ dos indivíduos são dependentes em suas atividades de vida diária (AVD's) e 46\% apresentam déficit cognitivo. No Brasil, aproximadamente $70 \%$ dos indivíduos não retorna ao trabalho e $50 \%$ são dependentes para realização das AVD's ${ }^{1-3}$.

Aproximadamente três quartos dos sobreviventes apresentam quadro de hemiparesia, e que, na maioria das vezes está acrescida de espasticidade, afetando 
negativamente o desempenho funcional e segurança em atividades dinâmicas como a marcha4.

Uma das alterações mais comuns em indivíduos pós AVC é o déficit no controle motor, impactando negativamente sobre a independência aumentando a propensão a quedas, sendo mais comum no primeiro ano. Estes pacientes diminuem a realização de atividade física, o que reflete em maior déficit funcional principalmente em habilidades como sentar, levantar e qualidade da marcha, gerando impactos na qualidade de vida desses indivíduos ${ }^{5}$.

Diferentes abordagens de reabilitação têm sido exploradas para tratar as sequelas de AVC. A Fisioterapia aquática é mais um recurso utilizado para estes pacientes, pois as propriedades físicas da água atuam sobre o corpo em imersão. Durante exercícios realizados em piscina terapêutica, o equilíbrio corporal é afetado tanto pela gravidade quanto pelo empuxo, e se essas forças não estiverem alinhadas sobre um eixo, o resultado é o efeito metacêntrico que promove um efeito rotacional e estimulação proprioceptiva podendo acionar estratégias de equilíbrio e endireitamento. Assim, esses estímulos frente aos desequilíbrios provocados pelo próprio meio líquido são compensados pelo aumento do tempo de resposta na água e permitem que o paciente tenha mais suporte e confiança para realizar as tarefas que em solo são mais desafiadoras ${ }^{6,7}$.

Entretanto, ainda são poucos os estudos que avaliaram aspectos relacionados à marcha em pacientes pós AVC, após treino funcional de marcha em ambiente aquático. 
Desta forma, o presente estudo tem como objetivo avaliar os aspectos relacionados à marcha, equilíbrio e velocidade em pacientes pós AVC após treino funcional em ambiente aquático.

\section{MÉTODO}

\section{Amostra}

Trata-se de um estudo prospectivo, série de casos, realizado na Associação de Assistência à Criança Deficiente $(A A C D)$ - Ibirapuera no período de janeiro de 2019 até dezembro 2019. O estudo teve aprovação do Comitê de Ética em Pesquisa (CAAE: 09437619.9.0000.0085).

A amostra foi constituída por pacientes com diagnóstico médico de AVC isquêmico, de ambos os sexos, idade entre 38 e 59 anos, diagnóstico funcional de hemiparesia à esquerda e/ou direita, com mais de um ano de lesão, que não apresentaram contraindicações de frequentar a piscina, capacidade de seguir instruções verbais para realização dos testes e dos exercícios, além de já ter realizado fisioterapia aquática, estar adaptado ao meio líquido e não estar realizando outro tipo de intervenção fisioterapêutica no momento do estudo.

Como critérios de exclusão: pacientes com instabilidade clínica, não deambuladores, déficits cognitivos que dificultem o entendimento das atividades propostas, patologias associadas que podem comprometer a execução da tarefa. 


\section{Procedimentos}

Foi agendada uma reunião com os participantes da pesquisa, explicado quais procedimentos seriam submetidos e após aceitarem em participar do estudo assinaram o Termo de Consentimento Livre e Esclarecido (TCLE). Os pacientes que apresentaram os critérios de inclusão, compuseram a amostra final e foram randomizados em dois grupos: grupo experimental (GE) - protocolo específico de treino de marcha no ambiente aquático e grupo controle (GC) - exercícios de fisioterapia aquática com objetivo de melhorar padrão de marcha. A randomização foi realizada através de 4 envelopes selados, opacos e não translúcidos. Esses envelopes foram embaralhados e repassados à equipe pesquisadora, contendo cada um o nome do próximo tratamento num cartão ( $G E$ ou $\mathrm{GC}$ ). À medida que os pacientes assinaram o TCLE o avaliador abria o envelope na sequência e alocava o paciente no grupo correspondente.

Os pacientes foram avaliados antes do iniciar os atendimentos em piscina terapêutica e imediatamente após o término das 12 semanas de atendimento (GE e GC) com os seguintes instrumentos em solo: Mini BESTest, foi idealizado para auxiliar na identificação do subsistema que pode ser o responsável pelo pobre equilíbrio funcional ${ }^{8}, 0$ Time Up and Go (TUG), foi realizado para avaliar a mobilidade funcional e o equilíbrio dinâmico ${ }^{9}$. O Teste de caminhada de 10 metros (TC10m): avaliou a performance e a velocidade da marcha em uma distância de 10 metros $^{10}$. Para a análise clínica dos testes TUG e TC10m, foi aplicado o 
G-Walk por meio de um sensor inercial sem fio aplicado ao paciente fornecendo dados para quantificar a eficácia da performance nos testes.

O programa de intervenção na Fisioterapia aquática para o GE quanto para o GC, foi de sessões de 35 minutos, 2 vezes por semana durante 12 semanas, em piscina terapêutica com a temperatura da água em torno de $34^{\circ} \mathrm{C}$. Nossa piscina possui vários níveis de profundidade sendo possível ajustar o espelho d'água para cada biotipo de paciente.

No GE foi aplicado o protocolo de atividades funcionais de marcha no ambiente aquático, todas as atividades foram realizadas com nível de imersão no processo xifoide sendo composto por:

1. Fase preparatória (alongamento passivo da cadeia posterior dos membros inferiores 3 séries de 30 segundos). Paciente sentado em um tablado com nível de imersão no processo xifoide, terapeuta realizou alongamento passivo.

2. Marcha anterior em linha reta durante 3 minutos.

3. Marcha anterior em linha reta com caneleira tradicional da marca Floty na região distal do membro inferior não acometido durante 3 minutos.

4. Marcha anterior em linha reta com tornozeleira de ginástica $1 \mathrm{~kg}$ da marca Slade Fitness na região distal do membro inferior parético durante 3 minutos.

5. Marcha posterior em linha reta durante 3 minutos. 
6. Marcha posterior em linha reta com caneleira tradicional da marca Floty contralateral ao membro inferior parético durante 3 minutos.

7. Marcha posterior em linha reta com tornozeleira de ginástica $1 \mathrm{~kg}$ marca Slade Fitness na região distal do membro inferior parético durante 3 minutos.

8. Marcha anterior em linha reta transpondo obstáculo (Step para Hidroginástica Hidrostep Fiore) durante 3 minutos.

9. Marcha anterior em linha reta transpondo Plataforma Modular Colorida Para Fundo De Piscina 2X5 da marca Fiore durante 3 minutos.

10. Marcha anterior contra turbulência da água gerada pelo aparelho da marca Água Viva durante 3 minutos.

11. Marcha lateral com resistência da Faixa elástica para exercícios (azul/média forte) Carci, colocada em terço distal da coxa, durante 3 minutos.

O GC realizou exercícios de alongamento muscular da cadeia posterior do membro inferior parético 3 séries de 30 segundos, paciente sentado em um tablado com nível de imersão no processo xifoide, terapeuta realizou alongamento passivo. Ativação muscular de tronco 3 séries de 12 repetições (paciente sentado em sela aberta realizando abdominais com apoio do terapeuta em quadril). Ativação muscular do membro inferior parético, 3 séries de 12 repetições (exercício 1 - paciente em postura ortostática com nível de imersão no processo xifoide apoia o membro inferior parético em uma plataforma modular colorida para fundo de 
piscina $2 \times 5$ da marca Fiore realiza movimento de subir na plataforma, exercício 2 - paciente em postura ortostática com nível de imersão em crista ilíaca ântero superior realiza movimento de agachamento). Treino de equilíbrio estático (paciente em postura ortostática em cima de uma superfície instável, cama elástica para hidroginástica Fiore, com nível de imersão no processo xifoide realiza alcance lateral de argolas (12 argolas para recreação submersa em piscina Fiore) e coloca no membro superior parético. Treino de equilíbrio dinâmico (paciente em postura ortostática com nível de imersão no processo xifoide tem que subir em superfície instável, plataforma modular colorida para fundo de piscina $2 \times 5$ da marca Fiore, andar até o final, realizar um giro e retornar ao início. Repetir o exercício por 3 minutos).

\section{Análise estatística}

A normalidade dos dados foi verificada por meio do teste ShapiroWilk. Em seguida, os dados foram analisados por meio do teste $\mathrm{t}$ de Student para amostras pareadas de pré e pós o protocolo, considerando o intervalo de confiança (IC) de 95\%, o nível de significância de $p<0,05$ e os dados apresentados em média.

\section{RESULTADOS}

A amostra foi composta por quatro indivíduos hemiparéticos pós-AVC, caracterizados na Tabela 1. 
Tabela 1. Caracterização da amostra.

Características

Gênero Masculino

Feminino

Diagnóstico AVE Isquêmico

Média de

Idade (anos)
GC

GE

1

2

1

2

2

59

46

$\mathrm{Na}$ Figura 1 foi observado melhora no equilíbrio funcional avaliado por meio do Mini BESTest pré e pós intervenção com diferença significativa, tanto no GE como no GC.

Na Tabela 2 observamos ganhos no pré e pós TC10m em ambos os grupos com diferença na duração e velocidade para o GE $(p=0,0109)$ e para o GC $(p=0,05653$ e $\mathrm{p}=0,03752$; respectivamente).

Analisando os resultados do TUG na maioria das médias das variáveis (Tabela 3), observamos resultados positivos no tempo da duração da fase da virada média e final no grupo experimental $(p=0,02822$ e $p=0,03191)$ e no tempo da duração da análise, duração da fase da virada média e velocidade máxima de rotação da virada média do grupo controle $(p=0,009, p=0,01201$ e $p=0,043)$. 
Figura 1. Análise das médias do Mini BESTest pré e pós intervenção.

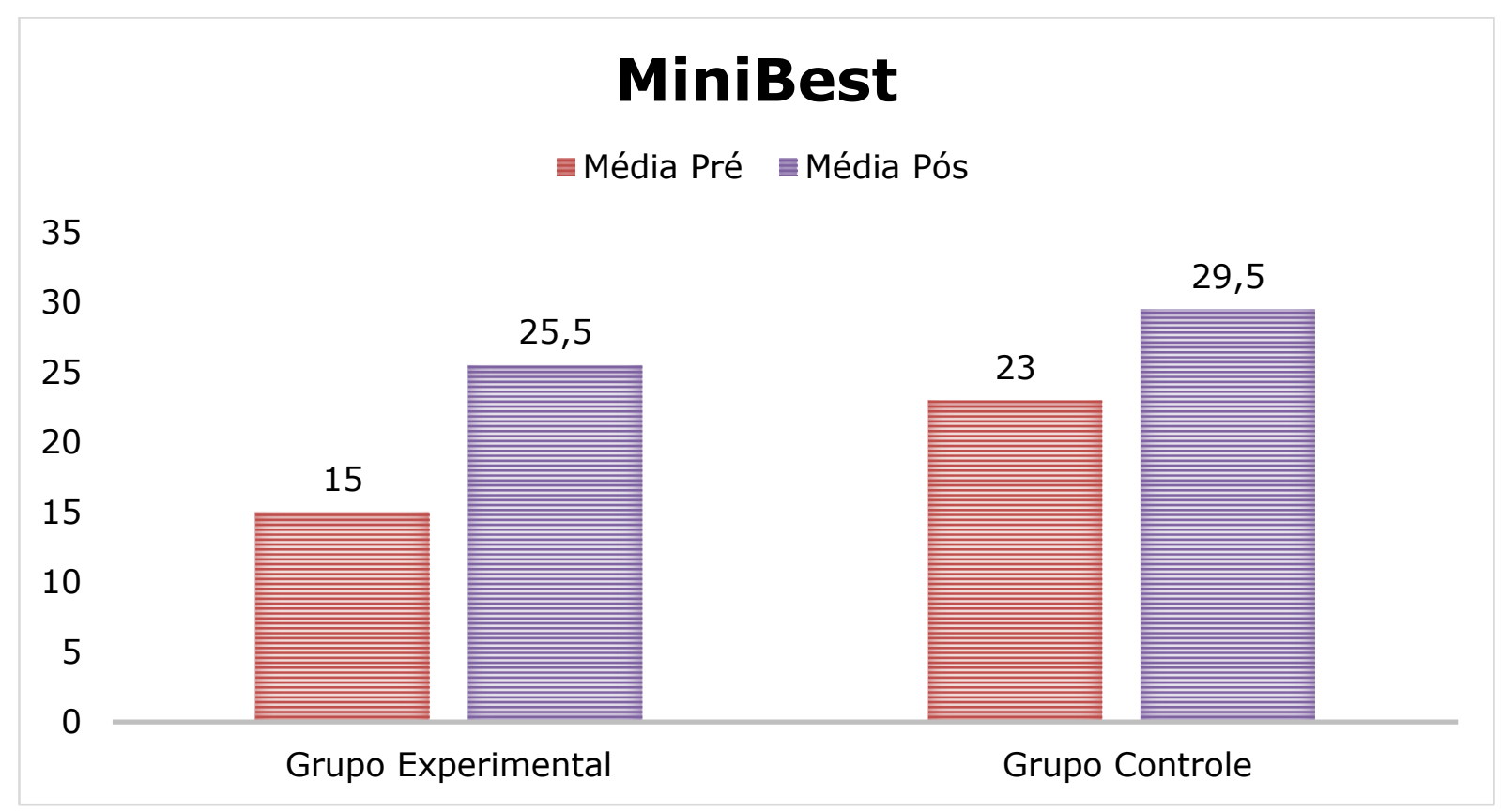

Grupo Experimental $p=0,015$; Grupo Controle $p=0,024 ; * p=$ valor $\leq 0,05$.

Tabela 2. Média pré e pós TC10M.

\begin{tabular}{cccccccc} 
& & \multicolumn{3}{c}{ Duração da Análise } & \multicolumn{3}{c}{ Velocidade } \\
\cline { 3 - 8 } & & Pré & Pós & & Pré & Pós & p \\
\cline { 3 - 8 } & & Média & Média & p-valor & Média & Média & p-valor \\
\hline \multirow{2}{*}{ TC10M } & \begin{tabular}{c} 
Experimental \\
\cline { 3 - 8 }
\end{tabular} & 20,90 & 16,95 & 0,1586 & 0,730 & 0,875 & $\mathbf{0 , 0 1 0 9}$ \\
\cline { 2 - 8 } & $\begin{array}{c}\text { Grupo } \\
\text { Controle }\end{array}$ & 17,50 & 15,55 & $\mathbf{0 , 0 5 6 5 3}$ & 1,023 & 1,175 & $\mathbf{0 , 0 3 7 5 2}$ \\
\hline
\end{tabular}


Tabela 3. Variáveis funcionais do TUG pré e pós intervenção.

\begin{tabular}{lcccccc} 
& \multicolumn{3}{c}{ GE } & \multicolumn{3}{c}{ GC } \\
\cline { 2 - 7 } & Pré & Pós & \multicolumn{3}{c}{ Pré } & Pós \\
\cline { 2 - 7 } & Média & Média & p-valor & Média & Média & p-valor \\
\hline Duração da Análise & 16,65 & 12,05 & 0,142 & 11,2 & 9,55 & $\mathbf{0 , 0 0 9}$ \\
\hline Sentado/Levantar (duração da fase) & 2 & 1,68 & 0,1053 & 10,8 & 12,25 & 0,3290 \\
\hline Em Pé/Sentar (duração da fase) & 1,18 & 1,08 & 0,0927 & 0,915 & 0,83 & 0,25 \\
\hline Virada Média (duração da fase) & 4,72 & 3,82 & $\mathbf{0 , 0 2 8 2 2}$ & 2,685 & 2,155 & $\mathbf{0 , 0 1 2 0 1}$ \\
\hline Virada Final (duração da fase) & 4,31 & 1,76 & $\mathbf{0 , 0 3 1 9 1}$ & 1,975 & 1,985 & 0,4922 \\
\hline $\begin{array}{l}\text { Virada Média (velocidade máxima } \\
\text { de rotação) }\end{array}$ & 123 & 128,5 & 0,084 & 151 & 173 & $\mathbf{0 , 0 4 3}$ \\
\hline $\begin{array}{l}\text { Virada Final (velocidade máxima de } \\
\text { rotação) }\end{array}$ & 123 & 166 & 0,167 & 155,5 & 169,5 & 0,147 \\
\hline
\end{tabular}

\section{DISCUSSÃO}

Este estudo teve como objetivo avaliar aspectos relacionados à marcha em pacientes pós AVC após treino funcional de marcha em ambiente aquático, notamos que após a aplicação do protocolo houve melhora no desempenho do equilíbrio e da velocidade.

O meio aquático aumenta a trajetória do centro de pressão durante o início da marcha, oferece resistência à execução do primeiro passo, impulso anteroposterior, e a força média aumenta enquanto se avança na água, sendo uma nova estratégia de tronco é evocada ao caminhar na água em comparação com o solo, sendo assim a água influencia na descarga do peso e oferece resistência ao movimento, desafiando o controle postural durante a antecipação e execução das fases do início da marcha. 
Condições estas que favorecem o treino de marcha, equilíbrio funcional e ativação muscular dos membros inferiores ${ }^{11}$.

O tempo de intervenção deste estudo foi de 2 vezes por semana durante 12 semanas totalizando 24 sessões, comparando com estudos na literatura foi possível notar que este tempo de intervenção foi suficiente para aprimorar 0 equilíbrio em pacientes pós $A V C$, demonstrando influência na velocidade da marcha e função dos membros inferiores ${ }^{12,13}$.

Após a intervenção realizada, pode-se notar melhora no equilíbrio por meio do Mini BESTest em ambos os grupos, mostrando como o exercício aquático exerce ação sobre o equilíbrio funcional em indivíduos pós AVC. Como se sabe o comprometimento do equilíbrio é um dos principais problemas físicos dos pacientes pós AVC que levam à limitação no desempenho das atividades da vida diária e participação na sociedade ${ }^{14}$, isso ocorre porque os déficits no equilíbrio e no controle da postura levam a uma alta incidência de quedas ${ }^{15,16}$.

Analisando as variáveis do TUG, os pacientes permaneceram dentro da faixa de classificação de independência parcial ou seja sem risco de quedas ${ }^{9,17}$, mas notamos melhora em diversas variáveis do TUG após a intervenção como: tempo da duração da fase da virada média e final no GE e no tempo da duração da análise, duração da fase da virada média e velocidade máxima de rotação da virada média do GC, indo de encontro com os resultados obtidos por outros estudos que mostraram 
diminuição de tempo no TUG após sessões de fisioterapia aquática com ênfase no equilíbrio e marcha ${ }^{18,19}$.

A melhora no desempenho do TUG ocorreu também por que 0 treino de marcha exige que os pacientes mantenham um bom controle de equilíbrio contra a força da gravidade, no entanto, pode ser difícil praticar essas reações em pacientes que apresentam comprometimentos de equilíbrio moderado a grave em solo, também é difícil desafiar e treinar a recuperação do equilíbrio apenas com exercícios em solo, devido ao possível medo de cair. Os exercícios na água incorporam a flutuabilidade, o que proporciona diminuição da carga dos membros inferiores podendo facilitar ou desafiar os movimentos horizontais e o equilíbrio. Praticar o equilíbrio em pé é frequentemente facilitado com o alinhamento postural apropriado usando a flutuabilidade da água, além de melhorar o equilíbrio diminuem o medo de cair facilitando a execução destes 7,20 embora não tenhamos medido o medo de cair acreditamos que por estar na água o paciente sente-se mais seguro, pois aumenta o tempo da possibilidade de resposta ao desequilíbrio devido às propriedades físicas da água.

Observamos um aumento na velocidade da caminhada tanto no GE quanto no GC, em solo, após intervenção no ambiente aquático. No GE foi utilizado caneleiras de peso no membro inferior hemiparético e marcha posterior. A velocidade de caminhada tem sido uma medida útil da recuperação funcional e da deambulação na comunidade em pessoas pós $A V C^{21}$. Foi mostrado um aumento da velocidade 
na marcha no ambiente aquático utilizando caneleiras de peso tanto no joelho como no tornozelo ${ }^{22}$. Outro estudo demonstrou que andar para trás aumenta a velocidade e diminui o padrão assimétrico da marcha em indivíduos com hemiparesia ${ }^{23}$.

A influência da imersão para as atividades em meio líquido depende da profundidade da mesma e consequentemente para a ativação muscular dos músculos estabilizadores. A melhora da execução da velocidade no pós-teste tanto no GE quanto no GC, pode estar relacionada à estabilidade, isto ocorre porque a sustentação de peso é gerada pelo empuxo no ambiente aquático sendo necessário aumentar a força propulsora para vencer a turbulência gerada pela resistência da água ${ }^{24}$.

A intervenção em grupo para pacientes com AVC, pode interferir nas percepções individuais em percepções coletivas e estas auxiliam no processo de recuperação e reabilitação pós AVC, acreditamos que este fator pode ter auxiliado na reabilitação, mas não podemos afirmar pois neste estudo não foi avaliado a variável atividade em grupo ${ }^{25}$.

A abordagem no meio líquido foi mais eficaz nos pacientes com AVC crônico, esses dados corroboram com este estudo, pois todos os pacientes já apresentavam, mais de um ano de lesão ${ }^{26}$.

As limitações deste estudo destacam-se a ausência de avaliação cega e o pequeno tamanho amostral. Apesar dos resultados positivos encontrados neste estudo, mais pesquisas são necessárias, abordando a melhora da marcha 
em pacientes pós AVC submetidos ao treino funcional de marcha em ambiente aquático, pois há uma escassez de protocolos evidenciando a eficácia de tais intervenções. Sugere-se que estes próximos estudos abordem um número maior de participantes.

\section{CONCLUSÃO}

A partir dos resultados obtidos neste estudo, pode-se inferir que o protocolo de treino funcional de marcha em ambiente aquático teve influência positiva sobre os aspectos relacionados à marcha em paciente pós AVC, em especial o equilíbrio e velocidade da marcha.

\section{REFERÊNCIAS}

1. Oliveira JRFD, Rodrigues SC, Santos MLL, Souza TA, Nascimento BB, França EMDM. Acidente vascular encefálico (AVE) e suas implicações na qualidade de vida do idoso: revisão bibliográfica. Temas em Saúde 2017; 17:283-99.

http://temasemsaude.com/wpcontent/uploads/2018/01/17419.pdf

2.Goldsteins LB, Bushnell CD, Adams RJ, Appel LJ, Braun LT, Chaturvedi $S$, et al. Guidelines for the Primary Prevention of Stroke A Guideline for Healthcare Professionals from the American Heart Association/American Stroke Association. Stroke 2011;42:517-84. https://doi.org/10.1161/STR.0b013e3181fcb238

3.Pontes-Neto OM. Sociedade Brasileira De Doenças Cerebrovasculares. Acidente vascular cerebral. Acesso em 29 de jan 2019. Disponível em: http://www.sbdcv.org.br/publica avc.asp

4. Matsumoto S, Uema T, Ikeda K, Miyara K, Nishi T, Noma T, et al. Effect of Underwater Exercise on Lower-Extremity Function and Quality of Life in Post-Stroke Patients: A Pilot Controlled Clinical Trial. J Altern Complement Med 2016;22:1-7.

https://doi.org/10.1089/acm.2015.0387

5.Tyson S, Connell L. The psychometric properties and clinical utility of measures of mobility in neurological conditions: a systematic review. Clin Rehabil 2009;23:1018-33.

https://doi.org/10.1177/0269215509339004 
6. Lee ME, Jo GY, Do HK, Choi HE, Kim WJ. Efficacy of Aquatic Treadmill Training on Gait Symmetry and Balance in Subacute Stroke Patients. Ann Rehabil Med 2017;41:376-86.

https://doi.org/10.5535/arm.2017.41.3.376

7. Oh S, Lim J, Kim Y, Kim M, Song W, Yoon B. Comparison of the effects of water- and land-based exercises on the physical function and quality of life in community-dwelling elderly people with history of falling: a single-blind, randomized controlled trial. Arch Gerontol Geriatr 2014;60:288-93. http://dx.doi.org/10.1016/j.archger. 2014.11.001

8.Franchignoni F, Horak F, Godi M, Nardone A, Giordano A. Using psychometric techniques to improve the Balance Evaluation Systems Test: the mini-BESTest. J Rehab Med 2010;42:323-31.

https://doi.org/10.2340/16501977-0537

9.Bischoff HA, Stähelin HB, Monsch AU, Iversen MD, Weyh A, von Dechend $M$, et al. Identifying a cut-off point for normal mobility: a comparison of the timed 'up and go' test in community-dwelling and institutionalised elderly women. Age Ageing 2003;32:315-20. https://doi.org/10.1093/ageing/32.3.315

10 .Watson MJ. Refining the ten-metre walking test for use with neurologically impaired people. Physiotherapy 2002;88:386-97. https://doi.org/10.1016/S0031-9406(05)61264-3

11.Marinho-Buzelli AR, Masani K, Rouhani H, Barela AM, Fernandes GTB, Verrier MC, et al. The influence of the aquatic environment on the center of pressure, impulses and upper and lower trunk accelerations during gait initiation. Gait Posture 2017;58:469-75. https://doi.org/10.1016/j.gaitpost.2017.09.008

12.Zhu Z, Cui L, Yin M, Yu Y, Zhou X, Wang $\mathrm{H}$, et al. Hydrotherapy vs. conventional land-based exercise for improving walking and balance after stroke: A randomized controlled trial. Clin Rehab 2015;30:58793. https://doi.org/10.1177/0269215515593392

13.Chu KS, Eng JJ, Dawson AS, Harris JE, Ozkaplan A, Gylfadottir S. Water-based exercise for cardiovascular fitness in people with chronic stroke: A randomized controlled trial. Arch Phys Med Rehabil 2004;85:870-4. https://doi.org/10.1016/j.apmr.2003.11.001

14.Ijmker $T$, Houdijk $H$, Lamoth $C$ J, Jarbandhan AV, Rijntjes D, Beek $\mathrm{PJ}$, et al. Effect of balance support on the energy cost of walking after stroke. Arch Phys MedRehabil 2013;94:2255-61.

https://doi.org/10.1016/j.apmr.2013.04.022

15.Park P, Noh J, Kim M, Lee L, Yang S, Lee W, et al. A comparative study of the effects of trunk exercise program in aquatic and landbased therapy on gait in hemiplegic stroke patients. Phys Ther Sci 2016;28:1904-8. https://doi.org/10.1589/jpts.28.1904

16.Park J, Roh H. Postural balance of stroke survivors in aquatic and land environment. Phys Ther Sci 2011;23:905-8. https://doi.org/10.1589/jpts.23.905

17.Noh DK, Lim JY, Shin HI, Paik NJ. The effect of aquatic therapy on postural balance and muscle strength in stroke survivors--a randomized controlled pilot trial. Clin Rehab 2008;22:966-76. https://doi.org/10.1177/0269215508091434 
18.Sá C, Pameira A. Results of a hydrotherapy program on balance, risk of falls, fear of falling and quality of life in older people. Physiotherapy $2015 ; 101:$ e1307.

https://doi.org/10.1016/j.physio.2015.03.1227

19. Chan K, Phadke CP, Stremler D, Suter L, Pauley T, Ismail F, et al. The effect of water-based exercises on balance in persons post-stroke: a randomized controlled trial. Top Stroke Rehab 2017;24:228-35. https://doi.org/10.1080/10749357.2016.1251742

20.Schimid A, Duncan PW, Studenski S, Lai SM, Richards L, Perera S, et al. Improvements in speed-based gait classifications are meaningful. Stroke 2007;38:2096-100.

https://doi.org/10.1161/STROKEAHA.106.475921

21.Nishiyori R, Lai B, Lee DK, Vrongistinos K, Jung T. The Use of Cuff Weights for Aquatic Gait Training in People Post-Stroke with Hemiparesis. Physiother Res Inter 2014;21:47-53. https://doi.org/10.1002/pri.1617

22. Yang Y, Yen J, Wang R, Yen L, Lieu F. Gait outcomes after additional backward walking training in patients with stroke: a randomized controlled trial. Saje Journals 2015;19:264-73. https://doi.org/10.1191/0269215505cr86oa.

23. Masumoto $\mathrm{K}$, Mercer JA. Biomechanics of human locomotion in water: An electromyographic analysis. Exerc Sport Sci Rev 2008;36:160-9. https://doi.org/10.1097/JES.0b013e31817bfe73

24.Rangel ESS, Belasco AGS, Diccini S. Qualidade de vida de pacientes com acidente vascular cerebral em reabilitação. Acta Paul Enferm 2013;26:205-12. http://dx.doi.org/10.1590/S010321002013000200016

25. Magalhães DRC, Matsui IM, Braga DM. Percepção da qualidade de vida de pacientes hemiparéticos pós-acidente vascular cerebral em um grupo de equilíbrio em ambiente aquático. Rev Bras Qual Vida 2019;11:e9493. https://doi.org/10.3895/rbqv.v11n3.9493

26.Van Duijnhoven HJ, Heeren A, Peters MA, Veerbeek JM, Kwakkel G, Geurts $\mathrm{ACH}$, et al. Effects of Exercise Therapy on Balance Capacity in Chronic Stroke: Systematic Review and Meta-Analysis. Stroke 2016;47:2603-10. https://doi.org/10.1161/STROKEAHA.116.013839 